\title{
Comparison of SWAN and WISE Menopausal Status Classification Algorithms
}

\author{
JANET M. JOHNSTON, Ph.D., ${ }^{1}$ ALICIA COLVIN, M.P.H., ${ }^{1}$ B. DELIA JOHNSON, Ph.D. ${ }^{1}$ \\ NANETTE SANTORO, M.D., ${ }^{2}$ SIOBÁN D. HARLOW, Ph.D., ${ }^{3}$ \\ C. NOEL BAIREY MERZ, M.D. ${ }^{4}{ }^{2}$ and KIM SUTTON-TYRRELL, Ph.D. ${ }^{1}$
}

\begin{abstract}
Background: Classification of menopausal status is important for epidemiological and clinical studies as well as for clinicians treating midlife women. Most epidemiological studies, including the Study of Women's Health Across the Nation (SWAN), classify women based on self-reported bleeding history.

Methods: The Women's Ischemia Syndrome Evaluation (WISE) study developed an algorithm using menstrual and reproductive history and serum hormone levels to reproduce the menopausal status classifications assigned by the WISE hormone committee. We applied that algorithm to women participating in SWAN and examined characteristics of women with concordant and discordant SWAN and WISE classifications.

Results: Of the 3215 SWAN women with complete information at baseline (1995-1997), 2466 $(76.7 \%)$ received concordant classifications (kappa $=0.52)$; at the fifth annual follow-up visit, of the 1623 women with complete information, $1154(72.7 \%)$ received concordant classifications (kappa $=0.57$ ). At each time point, we identified subgroups of women with discordant SWAN and WISE classifications. These subgroups, ordered by chronological age, showed increasing trends for menopausal symptoms and follicle-stimulating hormone (FSH) and a decreasing trend for estrogen $(p<0.001)$.

Conclusions: The WISE algorithm is a useful tool for studies that have access to blood samples for hormone data unrelated to menstrual cycle phase, with or without an intact uterus, and no resources for adjudication. Future studies may want to combine aspects of
\end{abstract}

\footnotetext{
${ }^{1}$ Department of Epidemiology, University of Pittsburgh, Pittsburgh, Pennsylvania.

${ }^{2}$ Division of Reproductive Endocrinology, Albert Einstein College of Medicine, Bronx, New York.

${ }^{3}$ Department of Epidemiology, University of Michigan, Ann Arbor, Michigan.

${ }^{4}$ Division of Cardiology, Department of Medicine, Cedars-Sinai Research Institute, Cedars-Sinai Medical Center, Los Angeles, California.

This work is based on the Study of Women's Health Across the Nation (SWAN), which has grant support from the National Institutes of Health, DHHS, through the National Institute on Aging, the National Institute of Nursing Research, and the NIH Office of Research on Women's Health (grants NR004061, AG012505, AG012535, AG012531, AG012539, AG012546, AG012553, AG012554, AG012495), and on The Women's Ischemia Syndrome Evaluation (WISE) Study, which was supported by contracts from the National Heart, Lung and Blood Institutes, Nos. N01-HV-68161, N01-HV-68162, N01-HV-68163, and N01-HV-68164, grants U0164829, U01 HL649141, and U01 HL649241, a GCRC grant MO1-RR00425 from the National Center for Research Resources, and grants from the Gustavus and Louis Pfeiffer Research Foundation, Denville, New Jersey, The Women's Guild of Cedars-Sinai Medical Center, Los Angeles, California, The Ladies Hospital Aid Society of Western Pennsylvania, Pittsburgh, Pennsylvania, and QMED, Inc., Laurence Harbor, New Jersey.
} 
the SWAN and WISE algorithms by adding hormonal measures to the series of bleeding questions in order to determine more precisely where women are in the perimenopausal continuum.

\section{INTRODUCTION}

$\mathrm{C}$ LASSIFICATION OF MENOPAUSAL STATUS is important for epidemiological and clinical research studies of midlife women, particularly for studies of menopausal symptoms, aging, and the role of reproductive hormones in various diseases. ${ }^{1-5}$ Menopausal status classification is also important to clinicians treating midlife women who need to be able to identify perimenopausal women in order to counsel them on ways to reduce their risk for chronic diseases, such as osteoporosis and heart disease, and to provide treatment options for menopausal symptoms, such as hot flashes and night sweats. ${ }^{6,7}$

Recently, considerable attention has been given to the development of staging systems for reproductive aging, ${ }^{8-11}$ but no single, agreed-upon standard exists for classification of menopausal status. Most epidemiological studies, including the Study of Women's Health Across the Nation (SWAN), make their determination based on questionnaire data about menstrual bleeding changes. ${ }^{12,13}$ Clinicians, on the other hand, are most likely to combine hormone measurements with bleeding history to determine if a woman is postmenopausal. ${ }^{14}$ Both of these approaches have drawbacks, with the former approach being likely to misclassify women who have irregular cycles prior to the transition, and the latter having unknown reliability as a result of the variability in hormone levels during the late perimenopause and postmenopause. A combination of both bleeding questions and hormone levels is intuitively appealing and has been proposed by the Stages of Reproductive Aging Workshop (STRAW) but is not frequently used, given the difficulty in determining how to appropriately categorize hormone levels in women who are undergoing the menopausal transition and the inherent within-woman between-cycle variability. 6,15

The Women's Ischemia Syndrome Evaluation (WISE) study ${ }^{16,17}$ addressed this problem by developing an algorithm using menstrual and reproductive history and results from serum hormone assays to reproduce the menopausal status classifications assigned by experts on the WISE hormone committee. In this paper, we extend the use of that algorithm by applying it to the cohort of women participating in SWAN and examining characteristics of women with concordant and discordant SWAN and WISE menopausal status classifications at various stages of the menopausal transition. We consider the relative usefulness of these two different types of menopausal status classification algorithms-one that relies on selfreports of menstrual bleeding and one that combines bleeding data and hormone measurements-for epidemiological and clinical studies.

\section{MATERIALS AND METHODS}

\section{Study population}

SWAN is a multicenter, multiethnic, longitudinal, community-based study designed to characterize the biological and psychosocial changes that occur during the menopausal transition. The SWAN cohort consists of 3302 women who were age $42-52$ years with at least one menstrual period in the previous 3 months when they were recruited into the SWAN study in 1995-1997. Women were recruited at seven study sites. The population at each site included Caucasian women and women from one designated minority (Boston, MA, Pittsburgh, PA, the Detroit, MI, area, and Chicago, IL, recruited African American women; Newark, NJ, recruited Hispanic women; Oakland, CA, recruited Chinese women; Los Angeles, CA, recruited Japanese women). Women with previous hysterectomy or bilateral oophorectomy were excluded from SWAN, as were women who took oral contraceptives (OC) or hormone therapy (HT) during the 3 months before the screening interview. Women were seen in the clinic at baseline and then annually for 5 years. The baseline and annual interviews included bleeding history, medical history, and a blood draw. For each follow-up visit, women who reported a hysterectomy, bilateral oophorectomy, or OC or HT use since their last visit were excluded from analyses related to that visit. All participants gave informed consent, and all study procedures were reviewed and approved by the 
institutional review boards of the participating institutions. A more detailed description of the SWAN population has been published previously. ${ }^{18,19}$

WISE is a four-center study designed to improve the diagnostic reliability of cardiovascular testing in the evaluation of ischemic heart disease in women. ${ }^{16}$ One of the major goals of the WISE study is to evaluate the influence of cyclical hormones, menopausal status, and blood reproductive hormone levels on cardiovascular physiology, symptoms, and diagnostic testing results. The population that served as the basis for the WISE menopausal status algorithm consisted of 515 women, aged 21-86 years, undergoing clinically ordered angiogram for suspected myocardial ischemia. Women who were taking OC or HT were excluded. The WISE study design called for only one participant contact, and the blood draw was not constrained to a specific time in the menstrual cycle. The WISE hormone committee reviewed and adjudicated menopausal status for each of 186 women who could not be definitely classified as postmenopausal, that is, previous bilateral salpingo-oophorectomy (BSO) or age $\geq 55$ years and no menses for 12 months. The WISE study population and WISE hormonal menopausal status algorithm have been previously described. ${ }^{17}$

\section{Measures of reproductive hormones}

Phlebotomy was performed in the SWAN study in the morning after overnight fast. Subjects were scheduled for venipuncture on days 2-5 after the onset of menses whenever possible. Women with blood draws that occurred outside of days 2-5 were included in these analyses because the WISE algorithm allows for blood draws at any time during the menstrual cycle.

All SWAN assays were performed on the ACS180 automated analyzer (Bayer Diagnostics Corporation, Tarrytown, NY) at the CLASS laboratory at the University of Michigan using a double-antibody chemiluminescent immunoassay with a solid-phase anti-IgG immunoglobulin conjugated to paramagnetic particles, antiligand antibody, and competitive ligand labeled with dimethylacridinium ester (DMAE). The folliclestimulating hormone (FSH) assay is a modification of a manual assay kit (Bayer Diagnostics) using two monoclonal antibodies ( $\mathrm{mAb}$ ) directed to different regions on the beta subunit, with a lower limit of detection (LLD) of $1.05 \mathrm{mIU} / \mathrm{mL}$. The estradiol $\left(E_{2}\right)$ assay modifies the rabbit anti$\mathrm{E}_{2-6}$ ACS-180 immunoassay to increase sensitivity, with an LLD of $1.0 \mathrm{pg} / \mathrm{mL}$. Duplicate $\mathrm{E}_{2}$ assays were conducted, with results reported as the arithmetic mean for each subject, with a coefficient of variation $(\mathrm{CV})$ of $3 \%-12 \%$. FSH assays were single determinations. ${ }^{20}$

The WISE study used the Diagnostic Products Corporation (Los Angeles, CA) coat-a-count kits for their FSH assay. $E_{2}$ was measured using steroid column extraction and subsequent assays. The CLASS laboratory that performed the SWAN $\mathrm{FSH}$ and $\mathrm{E}_{2}$ assays had previously determined that differences in sample collection and storage, as well as assay differences, caused systematically higher FSH readings in SWAN than in WISE. Therefore, the CLASS laboratory recalculated the previously published WISE FSH cutoff points of 10, 20, and 30 for menopausal classification and converted them to SWAN FSH measurements of 16,33 , and 50, respectively, in order to account for differences in the two studies' FSH measurements (Dan McConnell, University of Michigan, personal communication, 2004). The CLASS laboratory determined that no adjustments were necessary for the $E_{2}$ cutoff points.

\section{SWAN and WISE menopausal status algorithms}

Following the Massachusetts Women's Health Study, ${ }^{13}$ SWAN defines four menopausal status categories: premenopausal, $<3$ months of amenorrhea and no increase in menstrual irregularity in the past year; early perimenopausal, $<3$ months of amenorrhea with some increase in menstrual irregularity; late perimenopausal, between 3 and 11 months of amenorrhea; postmenopausal, 12 or more consecutive months of amenorrhea with no medical cause other than menopause. SWAN classifies women who have had a hysterectomy or a BSO as surgically menopausal, a separate category from women who are naturally postmenopausal.

The WISE hormonal classification committee, consisting of two reproductive endocrinologists, two clinical cardiologists, a statistician, and a nurse, categorized each woman in the WISE study into one of three status categories: premenopausal, perimenopausal, or postmenopausal. ${ }^{17}$ These classifications were based on age, body mass index (BMI), smoking history, history of a hysterectomy with or without bilateral or 
unilateral oophorectomy, cycle regularity, time since last menstrual period (LMP), and serum $\mathrm{FSH}$, luteinizing hormone $(\mathrm{LH}), \mathrm{E}_{2}$, estrone $\left(\mathrm{E}_{1}\right)$, and progesterone (PO) measurements. Using these committee-defined menopausal status classifications as the reference standard, the committee iteratively simplified the classification process into a decision tree by identifying the most important variables for status classification and establishing cutoff points that would reproduce the experts' status classification. The WISE algorithm requires age, time since last menstrual period, serum $\mathrm{E}_{2}$ and FSH values, and history of hysterectomy or BSO. Because SWAN separates sur- gical menopause from all other menopausal status classifications, we did not include the hysterectomy and BSO components of the WISE algorithm in our classification comparisons. Figure 1 illustrates the WISE algorithm for women who have not had a hysterectomy or BSO.

To evaluate agreement, we equated the SWAN premenopausal and postmenopausal classifications to the WISE premenopausal and postmenopausal classifications, respectively. Because the WISE classification scheme includes a single perimenopausal category and the SWAN classification scheme includes both early and late perimenopausal categories, we equated both the

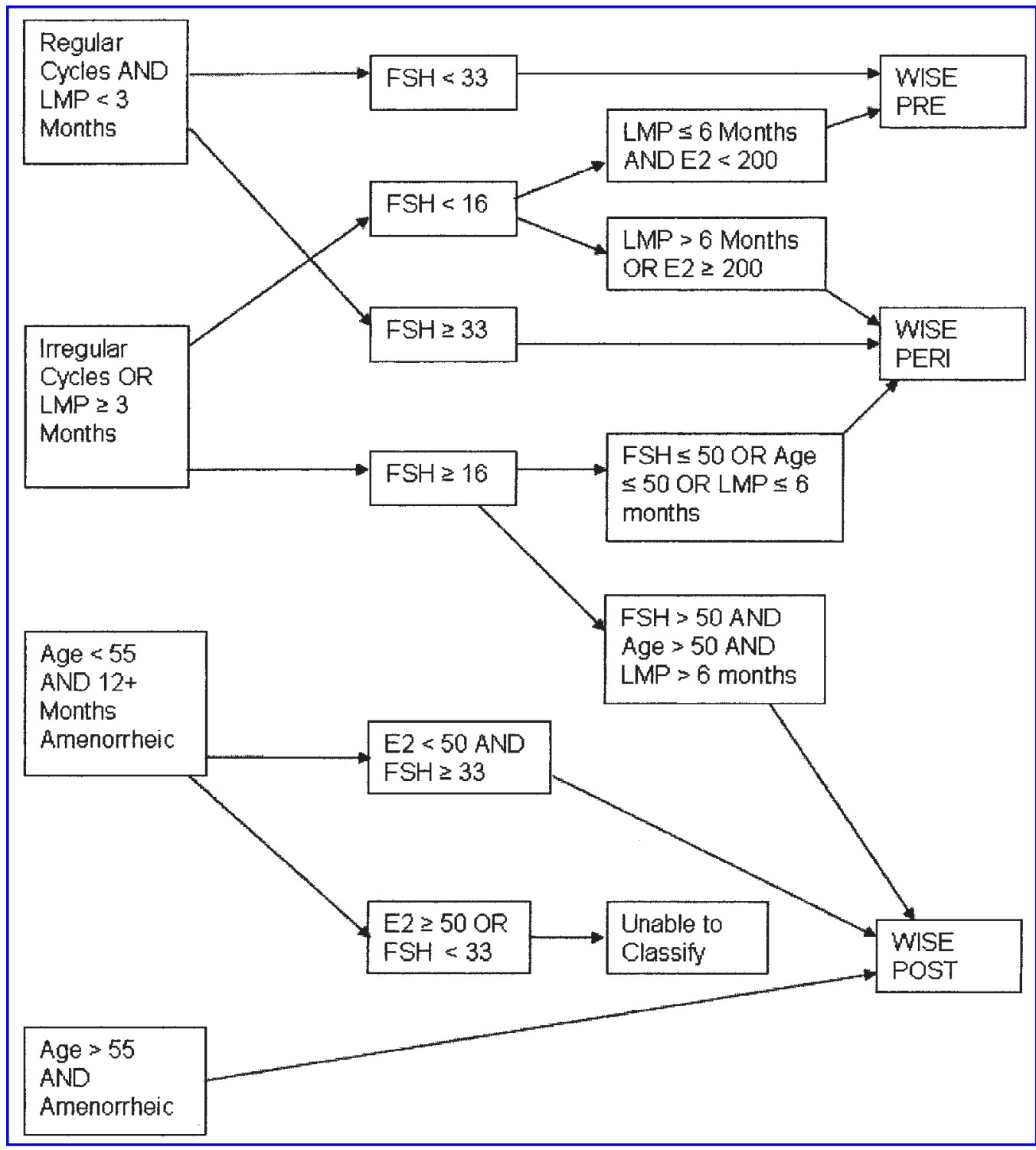

FIG. 1. WISE algorithm for women who have not had a hysterectomy or BSO. 
SWAN early and SWAN late perimenopausal classifications to the WISE perimenopausal classification.

We applied the two classification schemes to the SWAN population at baseline and at the fifth year of follow-up. Kappa statistics were computed to assess agreement. Sensitivity and specificity were not computed because there is no single, agreed-upon standard for menopausal classification. In order to examine differences between concordant and discordant women, we ordered the concordant and discordant subgroups by mean chronological age, which we used as a proxy for ovarian age. We then performed statistical tests for trend to detect whether there were linearly increasing or decreasing trends across the groups for presence of menopausal symptoms in the 2 weeks before the interview, for depressive symptomatology, and for $\mathrm{E}_{2}$ and FSH levels. We used the Cochrane-Armitage test for trend for dichotomous variable and an analogous nonparametric test based on Cuzick's Wilcoxon-type test for trend ${ }^{21}$ for continuous variables (STATA nptrend procedure). For follow-up visit 5, we limited this analysis to concordant and discordant subgroups with 50 or more women.

\section{RESULTS}

Based on SWAN's eligibility criteria, all women were classified as either premenopausal or early perimenopausal by SWAN at baseline. We applied the WISE hormonal algorithm to 3215 SWAN women (47\% Caucasian, 29\% African American, 8\% Chinese, 8\% Hispanic, and 8\% Japanese) who had age, hormone measures, and bleeding history information available at study entry. Of these women, 1724 (53.6 \%) were classified as premenopausal, and 1491 (46.4\%) were classified as early perimenopausal by the SWAN status classification algorithm (Table 1). The ma- jority of women $(88.5 \%)$ who were classified as premenopausal by SWAN were also classified as premenopausal by WISE; $11.5 \%$ were classified as WISE perimenopausal because they had an FSH value $\geq 33$. Of the women classified as early perimenopausal by SWAN, 63.0\% were also classified as perimenopausal by WISE, whereas 37\% were classified as premenopausal by WISE because of irregular cycles and an FSH value $<16$. The majority of women $(2466,76.7 \%)$ received concordant classifications with the SWAN and WISE algorithms based on information from the SWAN baseline visit. The kappa statistic for agreement was 0.52 , indicating moderate agreement.

To further explore discordance at baseline, we categorized women into the following four categories and ordered the groups by mean chronological age:

1. Concordant for premenopausal

2. Discordant: SWAN early perimenopausal and WISE premenopausal

3. Discordant: SWAN premenopausal and WISE perimenopausal

4. Concordant for perimenopausal

Looking across these subgroups of women, we found statistically significant linear trends (Cochrane-Armitage test for trend) for increased likelihood of reporting menopausal symptoms (hot flashes, cold sweats, and night sweats) in the 2 weeks before the baseline interview as we moved from the subgroups of women who were earliest in the menopausal transition (concordant for premenopausal) to those who were furthest along in the transition (concordant for perimenopausal) (Table 2). As expected, we also found significant linear trends for increasing mean FSH levels and decreasing mean estrogen levels as we moved from subgroups of women who were earlier to those who were later in the

Table 1. Cross-Tabulation of SWAN and WiSE Menopausal Status Classifications for 3215 SWAN Women at Baseline

\begin{tabular}{lccr}
\hline & WISE & WISE & Total \\
\hline Premenopausal & Perimenopausal & $1724(53.6 \%)$ \\
SWAN premenopausal & $1526(47.5 \%)$ & $198(6.2 \%)$ & $1491(46.4 \%)$ \\
$\quad 551(17.1 \%)$ & $940(29.2 \%)$ & $3215(100 \%)$ \\
$\quad$ perimenopausal & $2077(64.6 \%)$ & $1138(35.4 \%)$ & 3 \\
\hline
\end{tabular}


Table 2. Baseline Characteristics by SWAN/WISE Baseline Classifications ${ }^{a}$

\begin{tabular}{|c|c|c|c|c|c|}
\hline & $\begin{array}{c}\text { SWAN } \\
\text { premenopausal } \\
\text { /WISE } \\
\text { premenopausal } \\
(\mathrm{n}=1526) \\
\%\end{array}$ & $\begin{array}{c}\text { SWAN early } \\
\text { perimenopausal } \\
\text { /WISE } \\
\text { premenopausal } \\
(\mathrm{n}=551) \\
\%\end{array}$ & $\begin{array}{c}\text { SWAN } \\
\text { premenopausal } \\
\text { /WISE } \\
\text { perimenopausal } \\
(\mathrm{n}=198) \\
\%\end{array}$ & $\begin{array}{c}\text { SWAN early } \\
\text { perimenopausal } \\
\text { /WISE } \\
\text { perimenopausal } \\
(\mathrm{n}=940) \\
\%\end{array}$ & $\begin{array}{c}\mathrm{p} \text { value } \\
\text { for linear } \\
\text { trend }\end{array}$ \\
\hline \multicolumn{6}{|c|}{ Menopausal symptoms-any in 2 weeks before baseine interview } \\
\hline Hot flashes & 18 & 31 & 27 & 39 & $<0.0001$ \\
\hline Cold sweats & 8 & 14 & 14 & 15 & $<0.0001$ \\
\hline Night sweats & 23 & 34 & 32 & 37 & $<0.0001$ \\
\hline Feeling fearful & 16 & 23 & 16 & 24 & $<0.0001$ \\
\hline Nervous & 62 & 71 & 59 & 68 & 0.02 \\
\hline Heart racing & 28 & 35 & 26 & 35 & $<0.001$ \\
\hline $\begin{array}{l}\text { Frequent mood } \\
\text { changes }\end{array}$ & 46 & 59 & 42 & 56 & $<0.0001$ \\
\hline Irritable & 70 & 77 & 65 & 74 & 0.21 \\
\hline \multirow{2}{*}{$\begin{array}{l}\text { Depressed } \\
\quad(\text { CES-D } \geq 16)\end{array}$} & 21 & 31 & 22 & 26 & $<0.01$ \\
\hline & $\begin{array}{l}\text { Median } \\
(I Q R)^{\mathrm{b}}\end{array}$ & $\begin{array}{l}\text { Median } \\
(I Q R)\end{array}$ & $\begin{array}{l}\text { Median } \\
(I Q R)\end{array}$ & $\begin{array}{l}\text { Median } \\
(I Q R)\end{array}$ & $\begin{array}{l}\mathrm{p} \text { value } \\
\text { for trend }\end{array}$ \\
\hline Age (years) & $\begin{array}{c}45.5 \\
(43.7-47.5)\end{array}$ & $\begin{array}{c}45.5 \\
(43.5-47.2)\end{array}$ & $\begin{array}{c}47.4 \\
(45.0-49.3)\end{array}$ & $\begin{array}{c}47.4 \\
(45.3-49.4)\end{array}$ & $<0.01$ \\
\hline $\begin{array}{l}\text { Estrogen } \\
\quad(\mathrm{pg} / \mathrm{mL})\end{array}$ & $\begin{array}{c}60.8 \\
(39.3-90.3)\end{array}$ & $\begin{array}{c}68.1 \\
(42.5-99.6)\end{array}$ & $\begin{array}{c}26.9 \\
(18.8-42.6)\end{array}$ & $\begin{array}{c}42.7 \\
(26.3-83.3)\end{array}$ & $<0.01$ \\
\hline $\mathrm{FSH}(\mathrm{mIU} / \mathrm{mL})$ & $\begin{array}{c}13.5 \\
(10.0-18.6)\end{array}$ & $\begin{array}{c}11.1 \\
(8.5-13.2)\end{array}$ & $\begin{array}{c}46.5 \\
(38.9-62.6)\end{array}$ & $\begin{array}{c}28.2 \\
(19.6-48.9)\end{array}$ & $\begin{array}{l}<0.01 \\
<0.01\end{array}$ \\
\hline
\end{tabular}

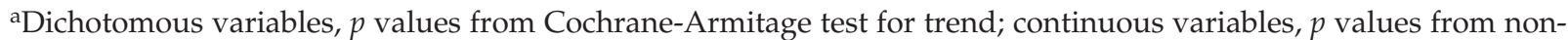
parametric test for trend (STATA, nptrend procedure).

${ }^{\mathrm{b}} \mathrm{IQR}=$ interquartile range.

menopausal transition (STATA nptrend procedure for continuous variables).

At the fifth follow-up interview, 1623 SWAN women had complete information, had not had a hysterectomy or BSO, and did not report HT use since the last study visit. Table 3 shows the cross-tabulation of SWAN and WISE menopausal status classification at follow-up visit 5 . Thirty-five women $(2.2 \%)$ who were identified as Unable to classify by the WISE algorithm were excluded from Table 3. Again, the major- ity of women $(1154,72.7 \%)$ received concordant classifications. The kappa statistic for agreement was 0.57 , again indicating moderate agreement.

Because of the large number of possible discordant classifications, we limited our analysis of concordant and discordant classification groups at follow-up visit 5 to the six groups that contained at least 50 women. The 80 women $(5.0 \%)$ with discordant classifications that did not fall into one of these six groups were excluded from

Table 3. Cross-Tabulation of SWAN and WisE Menopausal Status Classifications for 1588 SWAN Women at Follow-Up Visit 5a

\begin{tabular}{lcccc}
\hline & WISE & WISE & WISE \\
& Premenopausal & Perimenopausal & Postmenopausal & Total \\
\hline SWAN premenopausal & $87(5.5 \%)$ & $9(0.6 \%)$ & $0(0.0 \%)$ & $96(6.0 \%)$ \\
SWAN early perimenopausal & $336(21.2 \%)$ & $451(28.4 \%)$ & $0(0.0 \%)$ & $787(49.6 \%)$ \\
SWAN late perimenopausal & $17(1.1 \%)$ & $150(9.4 \%)$ & $53(3.3 \%)$ & $220(13.9 \%)$ \\
SWAN postmenopausal & $4(0.3 \%)$ & $15(0.9 \%)$ & $466(29.3 \%)$ & $485(30.5 \%)$ \\
$\quad$ Total & $444(28.0 \%)$ & $625(39.4 \%)$ & $519(32.7 \%)$ & $1588(100 \%)$ \\
\hline
\end{tabular}

aWomen were excluded from analysis if they reported HT use since last study visit or had a hysterectomy or BSO. 
this analysis. The groups were ordered by mean chronological age:

1. Concordant for premenopausal

2. Discordant: SWAN early perimenopausal and WISE premenopausal

3. Concordant: SWAN early perimenopausal and WISE perimenopausal

4. Concordant: SWAN late perimenopausal and WISE perimenopausal

5. Discordant: SWAN late perimenopausal and WISE postmenopausal

6. Concordant for postmenopausal

Again, we found statistically significant linear trends for increased likelihood of reporting menopausal symptoms (hot flashes, cold sweats, and night sweats) in the 2 weeks before the baseline interview as we moved from subgroups of women who were earlier in the menopausal transition to those who were later in the transition, with a noticeable flattening out at the $60 \%$ level for the reporting of hot flashes in the previous 2 weeks in the groups that were close to or had become postmenopausal (Table 4). Again, as expected, we found significant linear trends for increasing mean FSH levels and decreasing mean estrogen levels as we moved from subgroups of women who were earlier to those who were later in the menopausal transition.

\section{DISCUSSION}

In this study, we applied the WISE menopausal status classification algorithm, which includes the use of hormone levels, to women who had previously been classified using the SWAN menopausal status classification algorithm, which does not include hormone levels. We found that the two algorithms agreed for $77 \%$ of the SWAN women at baseline and for $73 \%$ of the SWAN women at the fifth annual follow-up visit. We further found that by combining the two algorithms, we could categorize women more finely. At baseline, we found highly significant

Table 4. Annual Follow-Up Visit 5 Characteristics by SWAN/WiSE Annual Follow-Up Visit 5 CLASSIFICATIONS $^{\mathrm{a}}$

\begin{tabular}{|c|c|c|c|c|c|c|c|}
\hline & $\begin{array}{c}\text { SWAN } \\
\text { premenopausal } \\
\text { /WISE } \\
\text { premenopausal } \\
(\mathrm{n}=87) \\
\%\end{array}$ & $\begin{array}{c}\text { SWAN early } \\
\text { perimenopausal } \\
\text { /WISE } \\
\text { premenopausal } \\
(\mathrm{n}=336) \\
\%\end{array}$ & $\begin{array}{c}\text { SWAN early } \\
\text { perimenopausal } \\
\text { /WISE } \\
\text { perimenopausal } \\
(\mathrm{n}=451) \\
\%\end{array}$ & $\begin{array}{c}\text { SWAN late } \\
\text { perimenopausal } \\
\text { /WISE } \\
\text { perimenopausal } \\
(\mathrm{n}=150) \\
\%\end{array}$ & $\begin{array}{c}\text { SWAN late } \\
\text { perimenopausal } \\
\text { /WISE } \\
\text { postmenopausal } \\
(\mathrm{n}=53) \\
\%\end{array}$ & $\begin{array}{c}\text { SWAN } \\
\text { postmenopausal } \\
\text { /WISE } \\
\text { postmenopausal } \\
(\mathrm{n}=466) \\
\%\end{array}$ & $\begin{array}{l}\mathrm{p} \text { value } \\
\text { for linear } \\
\text { trend }\end{array}$ \\
\hline \multicolumn{8}{|c|}{ Menopausal Symptoms-any in 2 weeks before annual follow-up visit 5 interview } \\
\hline Hot flashes & 27 & 30 & 44 & 60 & 60 & 60 & $<0.0001$ \\
\hline Cold sweats & 2 & 12 & 12 & 14 & 11 & 18 & $<0.001$ \\
\hline Night sweats & 24 & 36 & 38 & 46 & 36 & 42 & $<0.01$ \\
\hline Feeling fearful & 10 & 15 & 17 & 18 & 17 & 21 & $<0.01$ \\
\hline Nervous & 55 & 68 & 64 & 58 & 64 & 59 & 0.07 \\
\hline Heart racing & 25 & 28 & 24 & 26 & 23 & 28 & 0.67 \\
\hline $\begin{array}{l}\text { Frequent mood } \\
\text { changes }\end{array}$ & 39 & 49 & 40 & 39 & 42 & 43 & 0.56 \\
\hline Irritable & 75 & 74 & 65 & 60 & 55 & 60 & $<0.0001$ \\
\hline \multirow{2}{*}{$\begin{array}{l}\text { Depressed } \\
\quad(\mathrm{CES}-\mathrm{D} \geq 16)\end{array}$} & 20 & 19 & 17 & 15 & 26 & 23 & 0.07 \\
\hline & $\begin{array}{l}\text { Median } \\
(I Q R)^{\mathrm{b}}\end{array}$ & $\begin{array}{l}\text { Median } \\
(I Q R)\end{array}$ & $\begin{array}{l}\text { Median } \\
(I Q R)\end{array}$ & $\begin{array}{l}\text { Median } \\
(I Q R)\end{array}$ & $\begin{array}{l}\text { Median } \\
(I Q R)\end{array}$ & $\begin{array}{l}\text { Median } \\
(I Q R)\end{array}$ & $\begin{array}{l}\mathrm{p} \text { value } \\
\text { for trend }\end{array}$ \\
\hline Age (years) & $\begin{array}{c}49.1 \\
(48.0-50.6)\end{array}$ & $\begin{array}{c}49.3 \\
(48.2-50.8)\end{array}$ & $\begin{array}{c}50.3 \\
(48.9-52.0)\end{array}$ & $\begin{array}{c}51.2 \\
(49.3-53.0)\end{array}$ & $\begin{array}{c}53.2 \\
(51.5-54.8)\end{array}$ & $\begin{array}{c}53.6 \\
(51.8-55.2)\end{array}$ & $<0.01$ \\
\hline $\begin{array}{l}\text { Estrogen } \\
\quad(\mathrm{pg} / \mathrm{mL})\end{array}$ & $\begin{array}{c}50.6 \\
(27.9-81.2)\end{array}$ & $\begin{array}{c}54.0 \\
(31.2-109.2)\end{array}$ & $\begin{array}{c}36.1 \\
(18.7-83.1)\end{array}$ & $\begin{array}{c}22.8 \\
(14.1-45.2)\end{array}$ & $\begin{array}{c}11.3 \\
(8.6-15.1)\end{array}$ & $\begin{array}{c}13.0 \\
(9.4-18.7)\end{array}$ & $<0.01$ \\
\hline $\mathrm{FSH}(\mathrm{mIU} / \mathrm{mL})$ & $\begin{array}{c}12.6 \\
(9.6-18.7)\end{array}$ & $\begin{array}{c}11.7 \\
(8.9-15.1)\end{array}$ & $\begin{array}{c}33.5 \\
(21.1-57.8)\end{array}$ & $\begin{array}{c}72.7 \\
(39.1-96.2)\end{array}$ & $\begin{array}{c}90.2 \\
(74.8-116.4)\end{array}$ & $\begin{array}{c}88.5 \\
(65.6-115.9)\end{array}$ & $<0.01$ \\
\hline
\end{tabular}

aDichotomous variables, $p$ values from Cochrane-Armitage test for trend; continuous variables, $p$ values from nonparametric test for trend (STATA, nptrend procedure).

${ }^{\mathrm{b}} \mathrm{IQR}=$ interquartile range. 
differences in both estrogen and FSH levels between the two groups of women with discordant classifications, suggesting that hormone measurements may be useful when it is important to determine where women are in the continuum from premenopause to early perimenopause. At follow-up visit 5, we found noticeable steady increases in FSH values as we moved through the various concordant and discordant classification groups ordered according to mean chronological age. Again, this suggests that hormone levels may be useful for differentiating between premenopause and early and late perimenopause.

Menopausal transition stage is important in epidemiological and clinical research studies that include midlife women and in studies focused on understanding the natural history of the menopausal transition or the effect hormonal and other interventions have on that natural history. As the menopausal transition is a gradual and variable process, it is often difficult to determine a woman's menopausal status category. This study compared two proposed algorithms for classification and found moderate agreement between them. With no single agreed-upon standard for determining menopausal status, it is not surprising that two population-based algorithms that include different number of stages show some disagreement, particularly in regard to perimenopausal women.

The SWAN menstrually defined status categories are similar to those used by the Massachusetts Women's Health Study ${ }^{13}$ and included in World Health Organization (WHO) reports. ${ }^{22}$ The WISE algorithm was developed using a classification tree approach, with validation against classifications made by an expert committee. The WISE algorithm can be applied to women who may have stopped cycling for reasons unrelated to the menopause transition, such as hysterectomy, an important consideration for large epidemiological studies. Because SWAN's menstrually defined categories do not allow for menopausal status classification for women who have stopped cycling as a result of a hysterectomy, these women were not included in the comparison of the two algorithms.

The best method for determining menopausal status for a particular study or a particular woman will depend on characteristics of the study population or the individual, on the possibilities for data collection, and on the purpose of the assessment. For example, a study designed to test guidelines for physicians' counseling women on when to start monitoring bone mineral density (BMD) may need to depend on self-reported bleeding. A cardiovascular clinical trial evaluating the vascular effects of exogenous hormones may require precise measurements of endogenous hormone levels irrespective of bleeding history. Finally, a patient with severe menopausal symptomatology requiring treatment may warrant a combination of measurements of menstrual history, prospective menstrual charting, and hormone measurements to best assess her progress through the transition, whereas a mildly symptomatic patient will require a much less intensive assessment.

Two important questions researchers must consider are whether the additional information provided by hormone assays will improve status classification and whether the improvement is sufficient to warrant the added participant burden and cost. Although it has been shown that, on average, FSH levels are higher in women who are further along compared with those who are earlier in the transition, ${ }^{15,23}$ FSH levels for an individual woman may be extremely variable in the time leading up to the final menstrual period and, therefore, cannot be used in isolation to determine menopausal status. ${ }^{24}$ Estrogen levels also have been shown to be highly variable during the menopausal transition. ${ }^{15,23}$

Although it is not recommended that hormone levels alone be used to determine menopausal status, the approach used by the WISE algorithm combining hormone measurements with age and bleeding history may be well suited for study populations and clinical samples that include premenopausal and perimenopausal women who are not cycling regularly because of various health conditions. The WISE algorithm has the added benefit of categorizing women with a prior hysterectomy, a group that constitutes approximately $30 \%$ of women in this age group in the United States. ${ }^{25}$ It is not possible for the SWAN algorithm, which is based on bleeding history, to assign menopausal status to women who have undergone a hysterectomy. On the other hand, the SWAN algorithm has the advantage of differentiating between early and late perimenopause among women without hysterectomy.

Combining the two approaches may be useful when there is a need to determine status more precisely than is currently done by either 
SWAN or WISE. The STRAW proposed a staging system with seven stages ranging from early reproductive through late postmenopause. STRAW participants considered menstrual cyclicity, endocrinology, menopausal symptoms, fertility, and pelvic anatomy when developing their staging system for female reproductive aging; however, STRAW's staging criteria depend primarily on changes in bleeding characteristics and FSH levels. ${ }^{8} \mathrm{~A}$ recent multicohort evaluation of the STRAW criteria recommends inclusion of specific FSH values in defining menopausal stage. ${ }^{26}$

The classification tree used in the WISE algorithm was developed based on a small number $(n=32)$ of women considered perimenopausal by the WISE hormone committee, all of whom were undergoing clinically ordered angiograms for suspected myocardial infarction. ${ }^{17}$ Therefore, the decision points in the WISE algorithm may not be generalizable to more diverse populations.

Hormone levels may vary depending on the specific laboratory and assay. In our study, it was necessary to adjust the $\mathrm{FSH}$, but not $\mathrm{E}_{2}$, levels because of differences in the SWAN and WISE FSH assays.

\section{CONCLUSIONS}

The WISE algorithm is a useful tool for studies that have access to blood samples for hormone data unrelated to menstrual cycle phase, with or without an intact uterus, and no resources for adjudication. Our findings suggest that a menstrual status classification algorithm that relies solely on bleeding data can effectively distinguish among premenopausal, perimenopausal, and postmenopausal women. Future studies may want to combine aspects of the SWAN and WISE algorithms by adding hormonal measures to the series of bleeding questions to determine more precisely, at a population-based level, where women are in the perimenopausal continuum. Clinicians with access to more detailed information on their patients may be able to make more accurate assessments of menopausal status for individual women. Further work is needed to more precisely define the optimal bleeding and hormonal criteria for staging menopausal aging, building on these and other proposed approach. ${ }^{8-11}$

\section{ACKNOWLEDGMENTS}

We thank the study staff at each site and all the women who participated in SWAN and WISE. We thank Jessica Scottodiluzio for technical assistance in the preparation of this paper.

\section{REFERENCES}

1. Sowers MR, La Pietra MT. Menopause: Its epidemiology and potential association with chronic diseases. Epidemiol Rev 1995;17:287.

2. Oh JY, Barrett-Connor E, Wedick NM, Wingard DL. Endogenous sex hormones and the development of type 2 diabetes in older men and women: The Rancho Bernardo study. Diabetes Care 2002;25:55.

3. Kuller LH, Matthews K, Edmundowicz D, SuttonTyrrell K, Bunker C. Do changes in LDLc through menopause predict coronary and aortic atherosclerosis? Observations from the Healthy Women Study. Circulation 1999;99:1124.

4. Matthews KA, Kuller LH, Sutton-Tyrrell K, Chang YF. Changes in cardiovascular risk factors during the perimenopause and postmenopause and carotid artery atherosclerosis in healthy women. Stroke 2001;32:1104.

5. Matthews KA, Wing RR, Kuller LH, Meilahn EN, Plantinga P. Influence of the perimenopause on cardiovascular risk factors and symptoms of middleaged healthy women. Arch Intern Med 1994;154:2349.

6. Bastian LA, Smith CM, Nanda K. Is this woman perimenopausal? JAMA 2003;289:895.

7. Clinical challenges of perimenopause: Consensus opinion of The North American Menopause Society. Menopause 2000;7:5.

8. Soules MR, Sherman S, Parrott E, et al. Executive summary: Stages of Reproductive Aging Workshop (STRAW). Climacteric 2001;4:267.

9. Mitchell ES, Woods NF, Mariella A. Three stages of the menopausal transition from the Seattle Midlife Women's Health Study: Toward a more precise definition. Menopause 2000;7:334.

10. Taffe JR, Dennerstein L. Menstrual patterns leading to the final menstrual period. Menopause 2002;9:32.

11. Lisabeth LD, Harlow SD, Gillespie B, Lin X, Sowers MF. Staging reproductive aging: A comparison of proposed bleeding criteria for the menopausal transition. Menopause 2004;11:186.

12. Sternfeld B, Wang H, Quesenberry CP Jr, et al. Physical activity and changes in weight and waist circumference in midlife women: Findings from the Study of Women's Health Across the Nation. Am J Epidemiol 2004;160:912.

13. Brambilla DJ, McKinlay SM, Johannes CB. Defining the perimenopause for application in epidemiologic investigations. Am J Epidemiol 1994;140:1091.

14. Chiecchio A, Malvano R, Vignati G. The efficacy of hormone assays in the differential diagnosis of amen- 
orrhea and menopause. Clin Chem Lab Med 2000;38:971.

15. Burger HG, Dudley EC, Hopper JL, et al. Prospectively measured levels of serum follicle-stimulating hormone, estradiol, and the dimeric inhibins during the menopausal transition in a population-based cohort of women. J Clin Endocrinol Metab 1999;84:4025.

16. Merz CN, Kelsey SF, Pepine CJ, et al. The Women's Ischemia Syndrome Evaluation (WISE) study: Protocol design, methodology and feasibility report. I Am Coll Cardiol 1999;33:1453.

17. Johnson BD, Merz CN, Braunstein GD, et al. Determination of menopausal status in women: The NHLBI-sponsored Women's Ischemia Syndrome Evaluation (WISE) Study. I Womens Health 2004; 24:872.

18. Sowers MF, Crawford SL, Sternfeld B, et al. SWAN: A multicenter, multiethnic, community-based cohort study of women and the menopausal transition. In: Lobo RA, Kelsey J, Marcus R, eds. Menopause: Biology and pathobiology. San Diego: Academic Press, 2000:175.

19. Gold EB, Bromberger J, Crawford S, et al. Factors associated with age at natural menopause in a multiethnic sample of midlife women. Am J Epidemiol 2001;153:865.

20. Randolph JF Jr, Sowers M, Gold EB, et al. Reproductive hormones in the early menopausal transition: Relationship to ethnicity, body size, and menopausal status. J Clin Endocrinol Metab 2003;88:1516.

21. Cuzick J. A Wilcoxon-type test for trend. Stat Med $1985 ; 4: 87$.
22. Research on the menopause in the 1990s. Report of a WHO Scientific Group. World Health Organization Technical Report Series 1996;866:1.

23. Freeman EW, Sammel MD, Gracia CR, et al. Follicular phase hormone levels and menstrual bleeding status in the approach to menopause. Fertil Steril 2005; 83:383.

24. Burger HG. Diagnostic role of follicle-stimulating hormone (FSH) measurements during the menopausal transition-An analysis of FSH, oestradiol and inhibin. Eur J Endocrinol 1994;130:38.

25. Farquhar CM, Steiner CA. Hysterectomy rates in the United States 1990-1997. Obstet Gynecol 2002;99: 229.

26. Randolph J, Crawford S, Dennerstein L, et al. The value of follicle-stimulating hormone concentration and clinical findings as markers of the late menopausal transition. J Clin Endocrinol Metab 2006;91: 3034.

Address reprint requests to: Janet M. Johnston, Ph.D. Epidemiology Data Center Graduate School of Public Health University of Pittsburgh 127 Parran Hall, 130 DeSoto Street Pittsburgh, PA 15261

E-mail: johnston@edc.pitt.edu

\section{APPENDIX}

\section{The Study of Women's Health Across the Nation (SWAN)}

\section{Clinical Centers}

University of Michigan, Ann Arbor: MaryFran Sowers, PI; Massachusetts General Hospital, Boston, MA: Robert Neer, PI, 1994-1999, Joel Finkelstein, PI, 1999-present; Rush University, Rush University Medical Center, Chicago, IL: Lynda Powell, PI; University of California, Davis/Kaiser CA: Ellen Gold, PI; University of California, Los Angeles, CA: Gail Greendale, PI; University of Medicine and Dentistry-New Jersey Medical School, Newark, NJ: Gerson Weiss, PI, 1994-2004, Nanette Santoro, PI, 2004-present; University of Pittsburgh, Pittsburgh, PA: Karen Matthews, PI.

\section{NIH Program Office}

National Institute on Aging, Bethesda, MD: Marcia Ory, 1994-2001, Sherry Sherman, 1994-present; National Institute of Nursing Research, Bethesda, MD: Program officers.

\section{Central Laboratory}

University of Michigan, Ann Arbor, MI: Daniel McConnell (Central Ligand Assay Satellite Services). 


\section{Coordinating Center}

New England Research Institutes, Watertown, MA: Sonja McKinlay, PI, 1995-2001; University of Pittsburgh, Pittsburgh, PA: Kim Sutton-Tyrrell, PI, 2001-present.

Steering Committee: Chris Gallagher, Chair; Susan Johnson, Chair.

\section{The Women's Ischemia Syndrome Evaluation (WISE) Study}

\section{Clinical Sites}

University of Alabama at Birmingham, Birmingham, AL; University of Florida, Gainesville, FL; University of Pittsburgh, Allegheny Medical Center, Pittsburgh, PA.

\section{NIH Program Office}

National Heart, Lung and Blood Institute: George Sopko and Patrice Desvigne-Nikkens, Program Officers.

\section{Data Coordinating Center}

University of Pittsburgh, Pittsburgh, PA.

Clinical Coordinating Center and Reproductive Hormone Core Laboratory

Cedars-Sinai Medical Center, Los Angeles, CA.

Steering Committee: Noel Bairey Merz, Chair. 